\title{
Bibliothèque et Archives nationales du Québec et le milieu québécois de la documentation \\ Bibliothèque et Archives nationales du Québec and the \\ documentation landscape in Quebec \\ Bibliothèque et archives nationales du Québec y el medio quebequense de la documentación
}

\section{Lise Bissonnette}

Volume 54, numéro 2, avril-juin 2008

Topographie du Québec documentaire

URI : https://id.erudit.org/iderudit/1029309ar

DOI : https://doi.org/10.7202/1029309ar

Aller au sommaire du numéro

Éditeur(s)

Association pour l'avancement des sciences et des techniques de la documentation (ASTED)

ISSN

0315-2340 (imprimé)

2291-8949 (numérique)

Découvrir la revue

Citer cet article

Bissonnette, L. (2008). Bibliothèque et Archives nationales du Québec et le milieu québécois de la documentation. Documentation et bibliothèques, 54(2), 55-58. https://doi.org/10.7202/1029309ar
Résumé de l'article

De par la mission que lui confie sa loi constitutive, Bibliothèque et Archives nationales du Québec occupe une place à part au sein des milieux documentaires québécois. Elle s'attache ainsi à promouvoir la collaboration entre tous les intervenants de ces milieux, à soutenir des projets collectifs et à offrir ressources, appui et expertise dans une perspective d'harmonisation et de partage.
Tous droits réservés (C) Association pour l'avancement des sciences et des techniques de la documentation (ASTED) et Corporation des bibliothécaires professionnels du Québec (CBPQ), 2008
Ce document est protégé par la loi sur le droit d'auteur. L'utilisation des services d’Érudit (y compris la reproduction) est assujettie à sa politique d'utilisation que vous pouvez consulter en ligne. 


\title{
Bibliothèque et Archives nationales du Québec et le milieu québécois de la documentation
}

\author{
LISE BISSONNETTE \\ Présidente-directrice générale \\ Bibliothèque et Archives nationales du Québec
}

\section{RÉSUMÉ | ABstracts | RESUMEN}

De par la mission que lui confie sa loi constitutive, Bibliothèque et Archives nationales du.Québec occupe une place à part au sein des milieux documentaires québécois. Elle s'attache ainsi à promouvoir la collaboration entre tous les intervenants de ces milieux, à soutenir des projets collectifs et à offrir ressources, appui et expertise dans une perspective d'harmonisation et de partage.

\section{Bibliothèque et Archives nationales du Québec and the documentation landscape in Quebec}

The Bibliothèquè et Archives nationales du Québec's mission, as defined in its incorporating Act, plays an important and unique role within the province's information landscape. It endeavours to promote collaboration between stakeholders, to endorse cooperative projects and to offer ressources, support and expertise within a perspective of harmonization and sharing.

Bibliothèque et archives nationales du Québec y el medio quebequense de la documentación

De acuerdo a la misión que le ha confiado su propia Ley constitutiva, Bibliothèque et archives nationales du Québec (BAnQ) ocupa un lugar aparte al seno del medio quebequiense de la documentación. De esta manera, BAnQ se compromete a suscitar la colaboración entre todos aquellos que laboran en este campo, a promover los proyectos colectivos y a ofrecer en la medida de lo posible recursos, apoyo y pericia en una perspectiva de coherencia e uniformización de las prácticas y de cooperación.
\end{abstract}

L ONGTEMPS PARENTES PAUVRES de milieux culturels eux-mêmes astreints à une certaine frugalité, les bibliothèquès québécoises ont très tôt dans leur histoire développé des pratiques d'échange et de coopération pour tenter au mieux de satisfaire les demandes de leurs usagers, suivies dans cette voie par les centres d'archives créés dans les années 1970. Tout d'abord timides et dictées par des circonstances ponctuelles, limitées de plus par des moyens techniques et financiers modestes, ces collaborations ont pris dans la dernière décennie une dimension structurelle à l'échelle du territoire tout entier, portées par des technologies de l'information qui trouvent là un champ d'expression en expansion constante. Dans ce cheminement, au fil des ans, la Bibliothèque nationale du Québec, devenue en 2006 Bibliothèque et Archives nationales du Québec, s'est forgée une place à part au sein des milieux documentaires québécois, à la fois vitrine de l'expertise commune et catalyseur de projets collectifs de plus en plus nombreux.

\section{Une préoccupation institutionnelle ancienne}

À partir de 1967, alors qu'elle se voit dotée d'un statut de corporation qui lui confère une large autonomie, la Bibliothèque nationale du Québec assoit ses missions et mandats dans laction concrète et commence à se situer de façon singulière face aux bibliothèques québécoises. Elle semploie notamment à rassembler de façon exhaustive la totalité du patrimoine québécois publié et élabore de précieux outils de référence, telle la bibliographie nationale.

Il faut cependant attendre 1998 et la Loi sur la Grande bibliothèque du Québec pour voir le gouvernement du Québec confier officiellement à une institution documentaire une responsabilité spécifique face à un réseau encore en devenir. Ainsi, ce texte prévoit-il dans son Chapitre II, intitulé Missions et pouvoirs, que « la Grande bibliothèque a pour mission d'offrir un accès démocratique à la culture et au savoir et d'agir à cet égard comme catalyseur auprès des institutions québécoises contribuant ainsi à lépanouissement des citoyens». Plus particulièrement, il lui revient de "renforcer la coopération et les échanges entre les bibliothèques et de stimuler la participation québécoise à la bibliothèque virtuelle. » Elle doit de plus «susciter la coopération 
Il faut cependant attendre 1998 et la Loi sur la Grande bibliothèque du Québec pour voir le gouvernement du Québec confier officiellement à une institution documentaire une responsabilité spécifique face à un réseau encore en devenir.

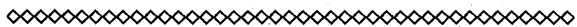

entre les bibliothèques publiques et les autres réseaux de bibliothèques et agir comme bibliothèque d'appoint pour l'ensemble'des bibliothèques publiques du Québec; développer et mettre à la disposition des bibliothèques publiques des services de soutien et d'expertise technique ; œuvrer à lélaboration d'un catalogue collectif virtuel et stimuler la participation des institutions documentaires au développement d'applications dans la bibliothèque virtuelle ». Précise, visionnaire et ambitieuse, cette loi pose les bases théoriques d'un véritable réseau documentaire québécois qui ne cessera dès lors de prendre rapidement de l'ampleur, notamment consolidé par deux décisions gouvernementales cruciales qui scelleront la force et loriginalité du modèle québécois.

En 2002 en effet, à la suite de la fusion entre la Bibliothèque nationale du Québec et la Grande bibliothèque du Québec, c'est à la " nouvelle BNQ » qu'il incombe de reprendre la mission définie en 1998 dans son intégralité, armée cette fois des ressources d'une bibliothèque nationale et d'une grande bibliothèque publique, et chargée de la responsabilité de coordonner une nouvelle instance formelle de coopération entre bibliothèques. Quatre ans plus tard, la fusion de la BNQ et des Archives nationales du Québec parachève cette dynamique douverture et de collaboration en confiant cette fois à Bibliothèque et Archives nationales du Québec (BAnQ) le soin de poursuivre les efforts entrepris, sur fond d'harmonisation croissante entre activités bibliothéconomiques et archivistiques.

La culture de la coopération et du partage requiert certes un état d'esprit mais aussi des moyens et des canaux permanents de communication qui la rendent consciente, solide et pérenne. C'est donc à travers un petit nombre de structures, certaines externes, d'autres faisant partie intégrante de son schéma institutionnel, que $B A n Q$ développe au quotidien son action au service des milieux documentaires québécois.

\section{La Table de concertation des bibliothèques québécoises}

En décembre 2002, le ministère de la Culture et des Communications du Québec décide de mettre sur pied une Table de concertation des bibliothèques québécoises placée sous la houlette de la présidente-directrice générale de la Bibliothèque nationale du Québec. Ce forum de dialogue et d'échange d'expertise regroupe des représentants de ce ministère, du ministère de l'Éducation, du Loisir et du Sport, de l'association Les Bibliothèques publiques du Québec, du Réseau BIBLIO du Québec, de la Conférence des recteurs et principaux des universités du Québec (CRÉPUQ) et du Réseau des services documentaires collégiaux (RESDOC). Les municipalités et les associations professionnelles concernées participent également à cette instance.

Dans une première phase de leurs travaux, les partenaires présents décident de réaliser une évaluation des différents réseaux de bibliothèques existants, d'en identifier les forces et les faiblesses afin de progresser vers une harmonisation et une mise à niveau de leurs ressources, méthodes et moyens. Il apparaît rapidement que lobjectif de renforcement des coopérations entre bibliothèques revêt une dimension technologique incontournable pour toute mise en réseau. Sur la base d'un sondage mené à léchelle du territoire et de cinq études expertes réalisées par un organisme indépendant, la Table de concertation présente, à l'issue de trois années de travail, des recommandations visant à orienter la mise à niveau technologique des bibliothèques publiques québécoises, et à accroître l'efficacité du traitement documentaire afin de mettre en place dès que possible un guichet unique ainsi qu'un catalogue collectif tout en facilitant le prêt entre bibliothèques pour le plus grand bénéfice des usagers. Pragmiatiques, fondées sur une approche réaliste et volontariste, ces recommandations sont entendues par le ministère de la Culture et des Communications qui, à l'automne 2006, décide de financer de façon récurrente la réalisation de ces objectifs. Parallèlement à ces réflexions, $B A n Q$ poursuit sur le terrain la concertation sur le catalogue collectif québécois et mène avec un petit nombre de bibliothèques pionnières l'expérimentation d'un modèle aujourd'hui opérationnel, dont le lancement a eu lieu à l'hiver 2008. Rarement résultats concrets auront été obtenus aussi rapidement, mais au-delà de ce succès, le grand mérite de la Table de concertation des bibliothèques québécoises aura été de créer un réflexe de dialogue et un climat de confiance jusqu'alors peu développés entre les représentants des différents milieux documentaires québécois. La prise de conscience de la similitude des problématiques auxquelles ces milieux sont confrontés et la volonté désormais acquise d'y apporter des réponses concertées ont enraciné une dynamique qui trouvera un nouveau champ d'expression dans la deuxième phase des travaux de la Table amorcée à la fin de l'année 2007. Cette seconde étape sera plus spécialement consacrée à la promotion des bibliothèques à travers l'amélioration de leur image, à l'étude des clientèles ainsi quà l'approfondissement des collaborations initiées entre les bibliothèques publiques et leur élargissement à d'autres secteurs, notamment celui des milieux éducatifs.

BAnQ nentend pas cependant limiter son action en faveur du renforcement du réseau documentaire québécois à cette mission de coordination des travaux de la 
Table de concertation des bibliothèques québécoises. Elle a conçu sa propre structure institutionnelle dans le but de proposer également des services spécialisés tant aux bibliothèques qu'aux milieux archivistiques.

\section{La Direction des services aux milieux documentaires de BAnQ}

Dès la création de la Grande bibliothèque du Québec en 1998, les réflexions liées à sa structure institutionnelle intègrent l'idée de mettre sur pied une direction spécifiquement vouée à répondre aux besoins des bibliothèques québécoises. La Direction des services aux milieux documentaires de BAnQ regroupe aujourd'hui six personnes dont la mission consiste à offrir des services de référence, des services conseil et du soutien professionnel. Elle s'attache également à coordonner divers projets coopératifs. Grâce à un extranet réservé aux bibliothèques et accessible sur le portail Internet de BAnQ, cette équipe de bibliothécaires aguerris est ainsi chargée de fournir à ses collègues information et documentation, à partir d'une collection spécialisée de plus de 7000 documents sur différents supports, traitant de questions aussi diverses que les pratiques de gestion, l'informatisation, l'architećture ou l'aménagement des bibliothèques.

Une fois inscrites à cet extranet, les bibliothèques peuvent aussi télécharger gratuitement les notices bibliographiques du catalogue Iris, s'enquérir des nouvelles acquisitions de BAnQ ou bénéficier du prêt entre bibliothèques.

En 2003, à l'initiative de BAnQ, a également été créé un Consortium dacquisition de ressources électroniques du Québec (CAREQ), qui permet de négocier des ententes communes avec des fournisseurs de ressources électroniques. BAnQ mène les travaux de négociation, assure la coordination des offres aux bibliothèques et leur suivi et participe financièrement à l'abonnement aux ressources acquises. Elle assume de plus le secrétariat $d u$ Comité de gestion du CAREQ qui regroupe des représentants des bibliothèques publiques québécoises.

BAnQ invite enfin les bibliothèques québécoises à intégrer sur leurs sites web des outils de référence originaux fort utiles à leurs usagers, tels « Romans@lire »ou le répertoire BREF qui recense plus de 1700 ressources de référence sur l'Internet.

L'extranet des bibliothèques, qui compte déjà plus de 400 bibliothèques inscrites, est appelé à étendre bien davantage encore son offre de services dans les années à venir afin de cimenter les acquis importants enregistrés depuis sa création.

Mais depuis 2006, un nouvel espace de coopération et de dialogue avec les milieux documentaires québécois s'est aussi ouvert pour BAnQ avec l'apport précieux de la Direction générale des Archives, née de la fusion entre la $\mathrm{BNQ}$ et les Archives nationales du Québec.
En 2002, à la suite de la fusion

entre la Bibliothèque nationale

du Québec et la Grande

bibliothèque du Québec, c'est à la

"nouvelle BNQ" qu'il incombe

de reprendre la mission définie

en 1998 dans son intégralité.

\section{Les services spécialisés en archivistique}

Déjà inscrite dans la Loi sur les archives adoptée en 1983 et favorisée par la régionalisation des Archives nationales opérée entre 1971 et 1980, l'offre de services spécialisés en archivistique et gestion documentaire a en effet pris une autre dimension avec la naissance de BAnQ.

Sappuyant sur des ressources technologiques accrues et bénéficiant de l'harmonisation des expertises réunies dans la nouvelle institution, ces services sadressent aux secteurs public et privé. BAnQ veille ainsi à ce que les archives historiques des organismes publics soient conservées, traitées, mises en valeur et rendues accessibles aux usagers. Son action touche quelque 142 ministères, organismes gouvernementaux et tribunaux à juridiction provinciale, ainsi que les administrations locales et régionales et les tribunaux locaux.

La Direction générale des Archives établit pour ce faire une panoplie sans cesse plus complète doutils de référence, politique de gestion des documents, calendriers de conservation, guides et formulaires qui constituent autant de points de repère utiles pour les milieux archivistiques. Elle supervise et coordonne également la préservation des archives privées sur leur lieu d’origine en réunissant notamment des tables de concertation régionales qui ont pour mission de dresser l'inventaire du patrimoine à sauvegarder et de partager les responsabilités de cette sauvegarde. Par ailleurs, BAnQ anime, dans le même esprit, un réseau de trente services d'archives privées agréés auxquels elle accorde un soutien financier.

Comme leurs collègues bibliothécaires, les archivistes québécois disposent d'un extranet spécialisé qui leur est réservé sur le portail Internet de BAnQ et qui, depuis son lancement l'an passé connait un succès croissant. 
Seule la mise en réseau de ressources documentaires numériques pourra permettre à l'ensemble des milieux documentaires québécois de préserver à long terme et de mettre en valeur leur patrimoine.

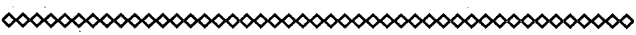

\section{Une ambition collective : la numérisation du patrimoine documentaire québécois}

Ces actions multiples, si essentielles soient-elles, se déploient cependant dans les limites d'une définition encore restrictive des milieux documentaires - bibliothèques publiques et centres d'archives - et peu représentative de l'extrême diversité de leurs intervenants. Seule la mise en réseau de ressources documentaires numériques pourra, dans un avenir que lon souhaite proche, permettre à l'ensemble des milieux documentaires québécois de préserver à long terme et de mettre en valeur leur patrimoine. Animée par une conscience aiguë de cette réalité, Bibliothèque et Archives nationales du Québec a pris l'initiative d’organiser deux journées de réflexion sur la numérisation culturelle au Québec en 2006. A la suite de ces échanges, il est apparu prioritaire de dresser un inventaire du patrimoine documentaire numérisé sur l'ensemble du territoire et d'explorer la possibilité de créer un réseau virtuel coopératif associant les principaux représentants des milieux documentaires québécois du domaine de la culture et de l'éducation. BAnQ s'est donc engagée à l'automne 2007 , en partenariat avec la Société des musées québécois, dans l'étude de ces ressources et des voies de conceptualisation de cette vitrine documentaire virtuelle.

Institution atypique regroupant selon un schéma totalement original une bibliothèque nationale, des archives nationales, une grande bibliothèque publique et un espace virtuel unique de documentation et de services, BAnQ ne peut, par nature, concevoir son engagement quotidien au service d'un accès sans cesse plus démocratique à la culture et au savoir sans interagir de façon permanente avec l'ensemble des milieux documentaires québécois. Il n'y a dans cette approche nulle concession à l'air du temps, à une incontournable logique des réseaux, mais bien la conviction profonde d'une urgence à créer des réflexes de partage vitaux pour la sauvegarde et le rayonnement de la culture québécoise. $\odot$
LA LIBRAIRIE MERCIER a pour objectif de faire tout son possible afin de simplifier votre travail.

Notre expérience nous permet d'effectuer des recherches fréquentes pour vous, et ainsi. réduire le nombre de vos commandes et vous sauver du temps.

Depuis 1952, nous diesservons les institutions dienseignement et de recherche, telles que les bibliothèques municipales, scolaires, prouinciales, féderales et d'hôpltaux.

Nous comptons avoir le privilège de bien vous servir très bientôt.

LIBRAIRIE MERCIER librairie agréée, 40, St-Joseph, Ste-Thérèse, Qc J7E 3L6 Téléphone : (450) 435-0581

Télécopieur : (450) 430-1584

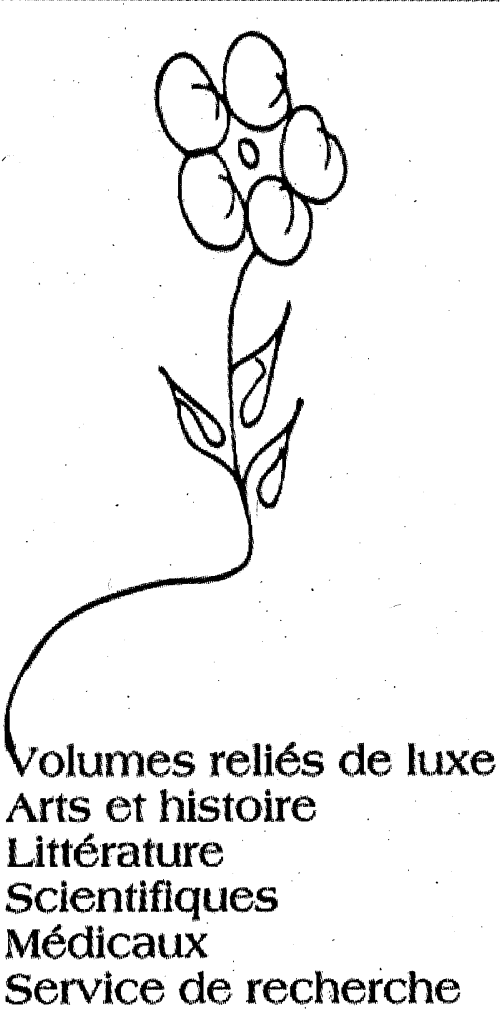

